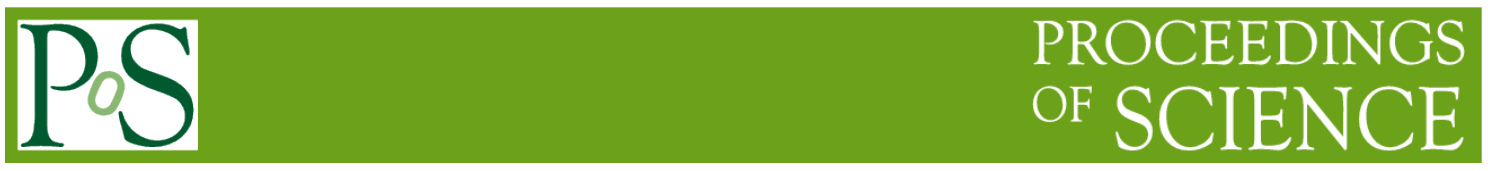

\title{
The Shashlik Calorimeter, a LYSO/W plate Calorimeter for Precision EM Calorimeter in the High Luminosity LHC environment
}

\section{B. Cox ${ }^{1}$}

University of Virginia

Charlottesville, VA 22904 USA

E-mail: bbc2x@virginia.edu

The future of HEP collider experiments for the next couple of decades may well require detectors that can withstand successively higher and higher luminosities. It has already become clear in the Atlas and CMS experiments at the LHC that major upgrades to detector components will be required to allow them to withstand integrated luminosities and pileup of $3000 \mathrm{fb}^{-1}$ by the 2025-30 period. Calorimeters are essential component of each of the major LHC detectors and the question is whether a calorimeter based on optical techniques such as the Shashlik technique can be made radiation hard enough to be a viable option for $3000 \mathrm{fb}^{-1}$ and beyond. This paper describes studies done for the CMS calorimeter upgrade.

38th International Conference on High Energy Physics

3-10 August 2016

Chicago, USA

${ }^{1}$ Speaker 


\section{Introduction}

It became obvious (due to the work of the CMS FCAL group, predecessor of the Shashlik group) in the time period 2010-2012 that the PbWO(4) endcaps would not survive to the stated goal of an integrated luminosity of 3000 inverse $\mathrm{fb}$ for the Phase II operation of the CMS detector in the decade of the 2020's. Fig. 1 shows the actual experience to date of the CMS endcap transparency loss and Fig. 2 shows a simulation of the loss of Higgs mass resolution as a function of eta and integrated luminosity.

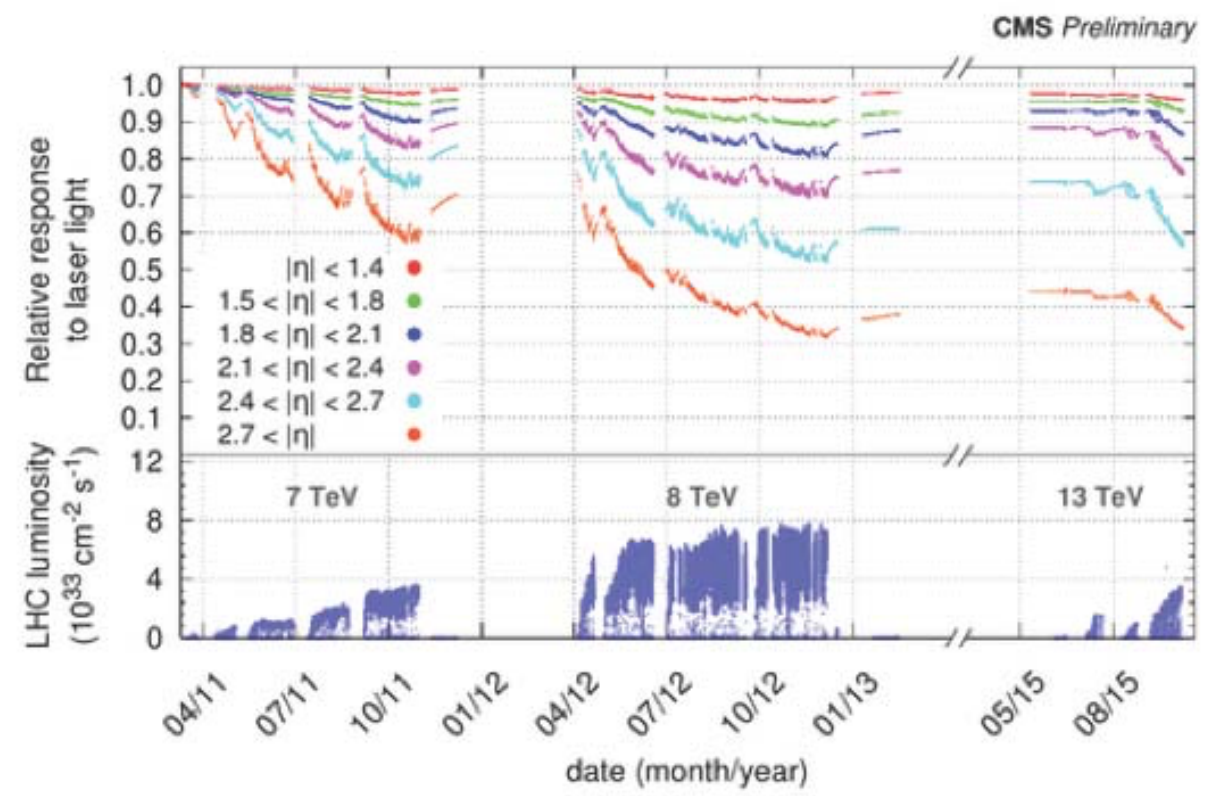

Fig.1 Transparency loss in the CMS endcap calorimeter as a function of eta and luminosity.

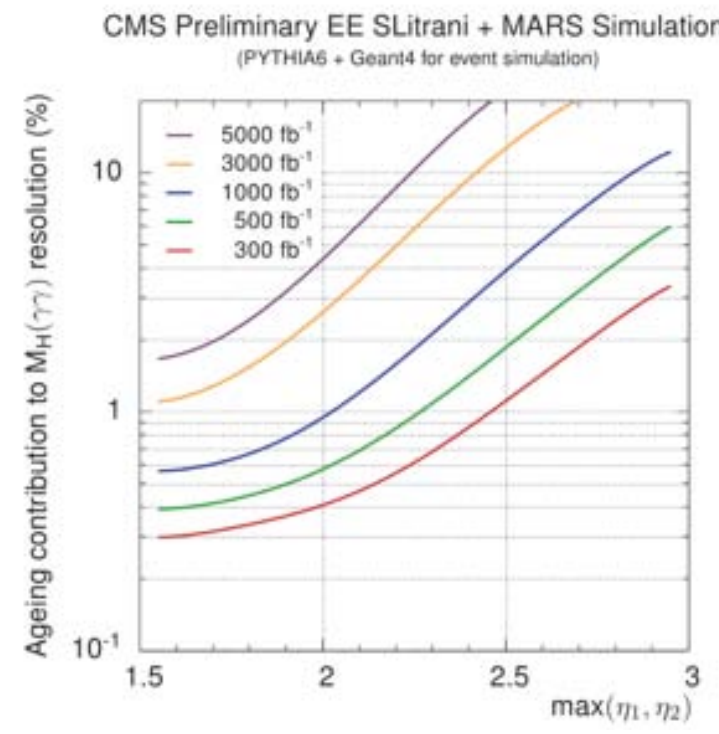

Fig. 2 aging contribution to the Higgs mass resolution. 


\section{Shashlik Strategy for Dealing with Radiation and Pileup issue}

Finding ways to lessen these radiation effects by hardening the various parts of the calorimeter system was assigned to the CMS FCAL group with the charge to

- do as well or better in photon (and diphoton) resolution as the undamaged $\mathrm{PbWO}(4)$ electromagnetic endcap.

- do no worse than the present hadronic HCAL endcap on hadrons.

Starting with this charge, three different options were investigated in a "downselect" process that resulted in two options selected for a final downselect:

The Shashlik option (light based option)

The HGC option (electrical based option)

This paper will discuss the Shashlik option. The requirements of the endcap calorimeter imposed by this charge are:

- Radiation hardness of calorimeter components to integrated fluxes of a few x 1015/cm2

- Detector speed (fast scintillation response of detector material and waveshiftes)

- Ability to deal with pileup (minimize shower size with high density of detector material)

- Sufficient detector element light output to minimize stochastic contribution to resolution

- Avoidance of major systematic constant term contributions so as to maintain $1 \%$ or better.

\section{The Shashlik Module}

The configuration of the individual Shashlik calorimeter elements that will address all the criteria listed above is shown in Fig. 3 below:

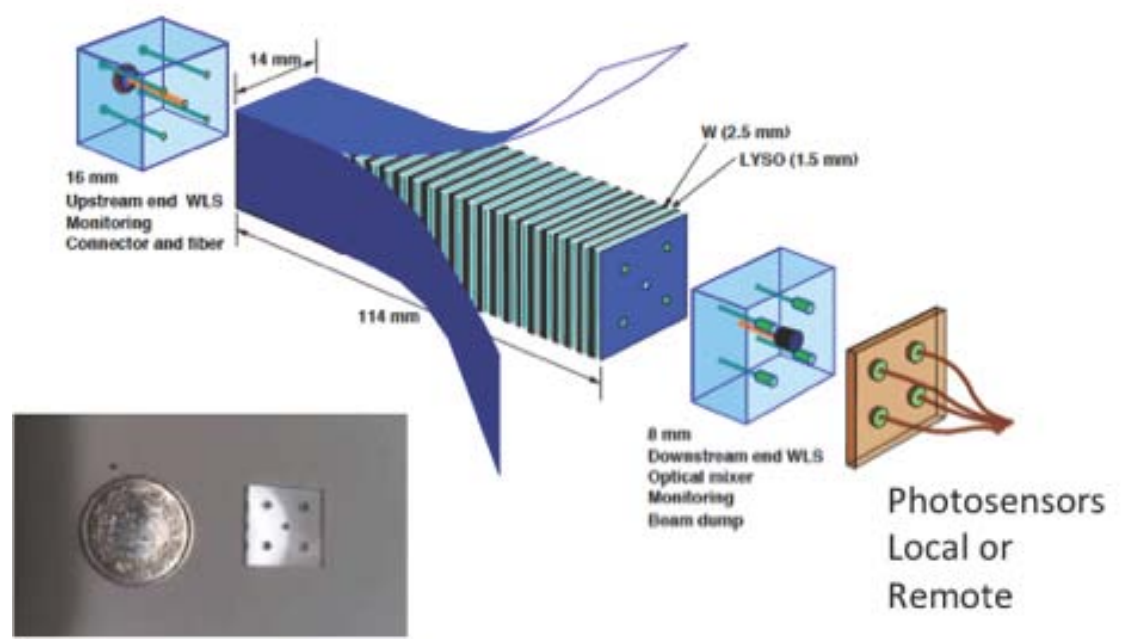

Fig. 3: The Shashlik Calorimeter element. Note the size of each of the 28 tungsten and 29 LYSO plates comprising the bulk of the calorimeter module as represented in the inset in the lower right corner of Fig. 3. 
Each plate has the approximate area of a $1 / 2$ Swiss franc piece. There are five holes in each plate; four for radiation hard waveshifter capillaries to transport the light from the showers in the LYSO crystal plates to the photosensors and one for a central fiber to inject calibration light to the elements of the modules.

Dealing with radiation hardness and high density of flux is accomplished by 1) use of dense materials leading to small Moliere radius of the showers, 2) radiation hard materials, 3) short optical path lengths for photons in the device to minimize effect of loss of transmission, 4) radiation resistant, small pixel photosensors placed in sheltered positions. The technologies chosen to achieve these features in the Shashlik element are:

- LYSO(Ce) that has high brightness, slow radiation degradation, and blue-violate emission spectrum [1]

- Capillaries [2] that are sealed and replaceable and that have liquid cores that can accommodate various WLS materials, making spectral matching straightforward.

- Photosensors made with using either GaInP devices [3] if positioning of the photosensors in a high radiation area is required or cooled, small pixel SiPM photosensors if a more protected location for placement is available [4].

Using the Shashlik module configuration, survival to $3000 \mathrm{fb}-1$ and beyond appears to be feasible.

\section{The Shashlik Endcap Calorimeter Configuration}

The arrangement of 30,500 of these elements configured in to one CMS endcap as shown in Fig. 4a and b. This configuration has been simulated to have the performance under high integrated radiation doses as shown in Fig. $5 \mathrm{a}$ and $\mathrm{b}$.
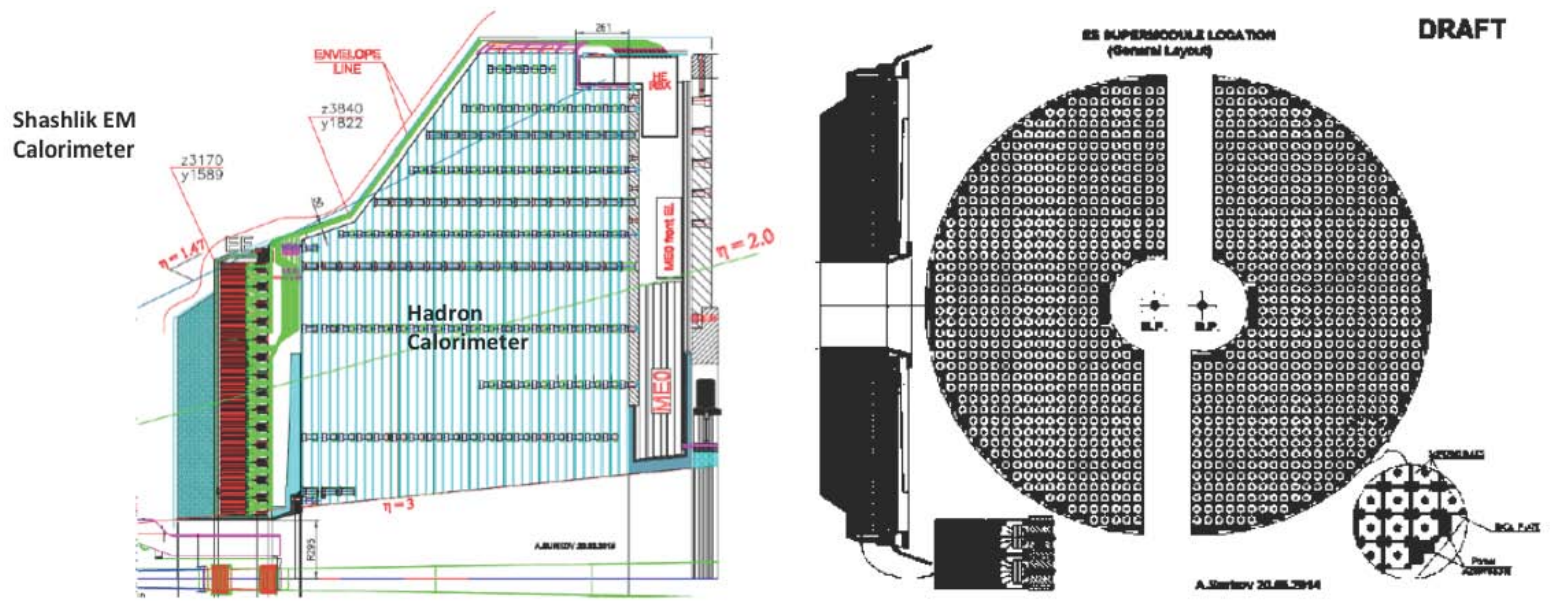

Fig 4a) Elevation View of the Shashlik Fig. 4b) View of the 30,500 elements element arrangement (plus from the interaction point backing hadron calorimeter) 

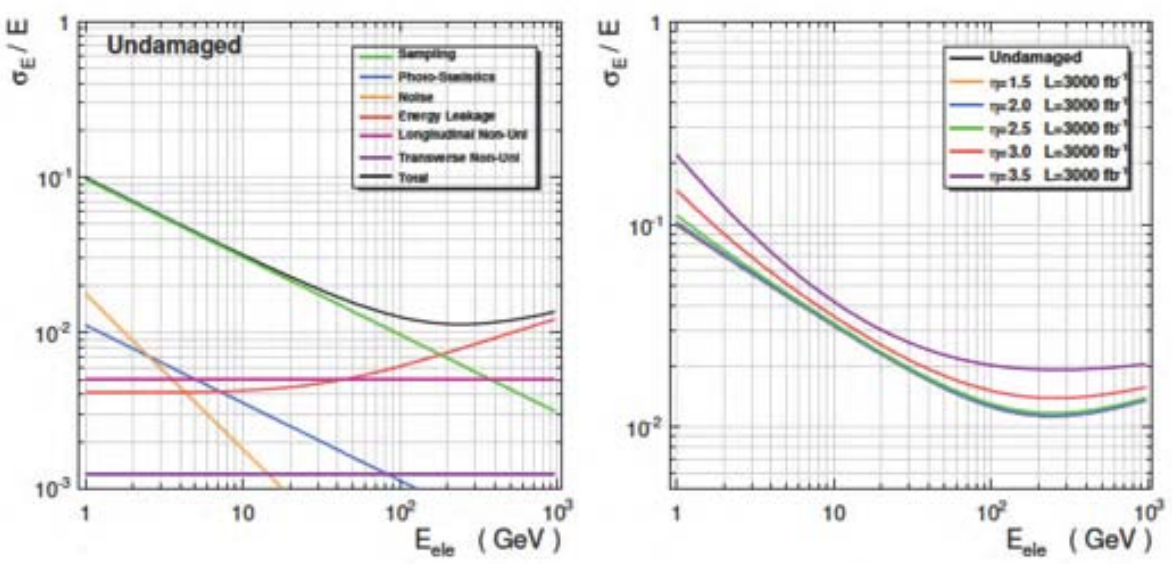

Fig. 5a) Undamaged resolution achievable Fig. 5b) Resolution as a functionwith the Shashlik calorimeter design of eta after $3000 \mathrm{fb}$.

\section{The Shashlik Test Beam Studies}

The Shashlik design resolution has been tested with electron beam in the CERN H4 test beam using a $4 \times 4$ matrix of elements constructed as proposed in Fig. 1, with module construction consisting of $28 \mathrm{~W}$ plates and 29 LYSO crystal plates as proposed for the Shashlik endcap. The four capillaries per module were rad hard Quartz (Polymicro QA): OD:ID = $1 \mathrm{~mm}: 0.4 \mathrm{~mm}, 180 \mathrm{~mm}$ long, filled with DSB1 waveshifting fluid that shifted the $420 \mathrm{~nm}$ LYSO emission light to $500 \mathrm{~nm}$ for transport principally in the quartz outer cylinder to $10 \mu \mathrm{m}$ pixel SiPMs. The measured $200 \mathrm{GeV}$ electron peaks achieved using either Y11 multiclad fibers or DSB1 liquid-filled capillaries for light transport are compared in Fig. 6a) and resolution versus beam energy from both light transport methods in Fig. 6b.
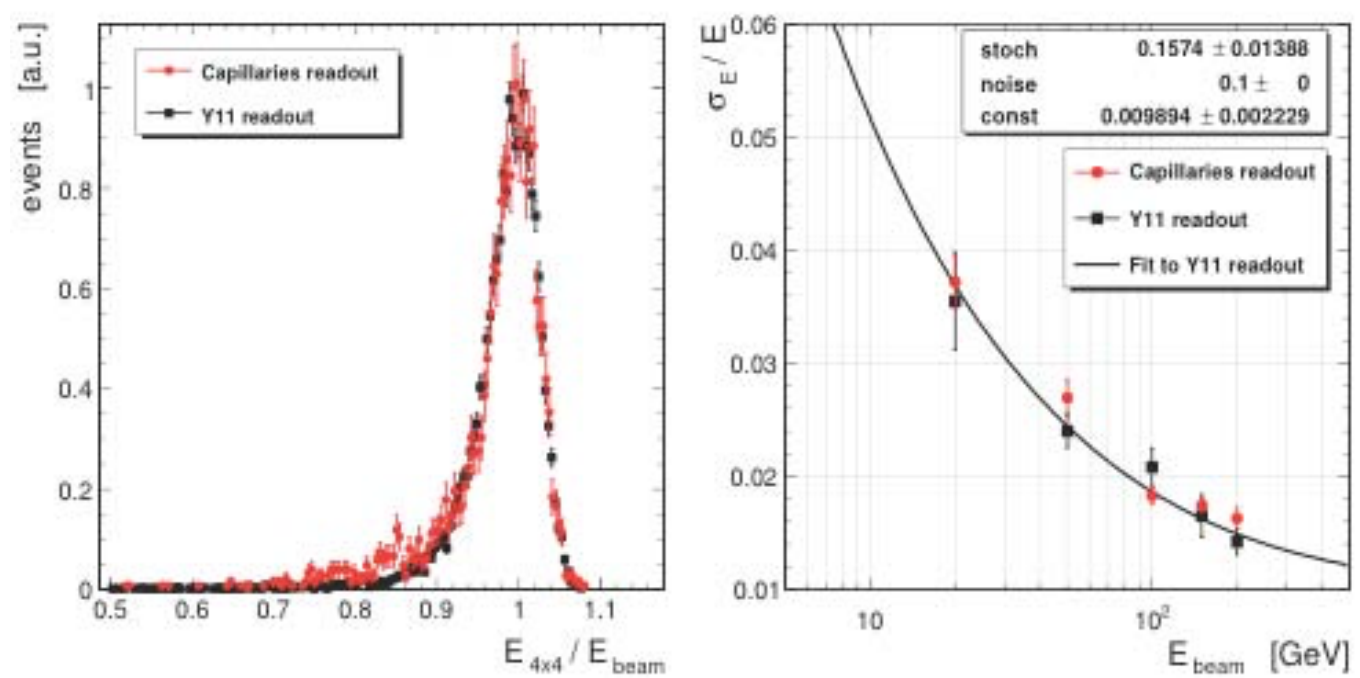

Fig. 6a) The $200 \mathrm{GeV}$ electronic peak as Fig. 6b) EM resolution as obtained with obtained using either capillaries capillaries or Y11 is essentially or Y11 fibers the same. 
It should be noted that the resolutions do not yet have corrections for the saturation effects that $200 \mathrm{GeV}$ electrons cause in the $10 \mu \mathrm{m}$ pixel SiPMs. No visible difference is seen between radiation hard capillaries and the Y11 fiber readout.

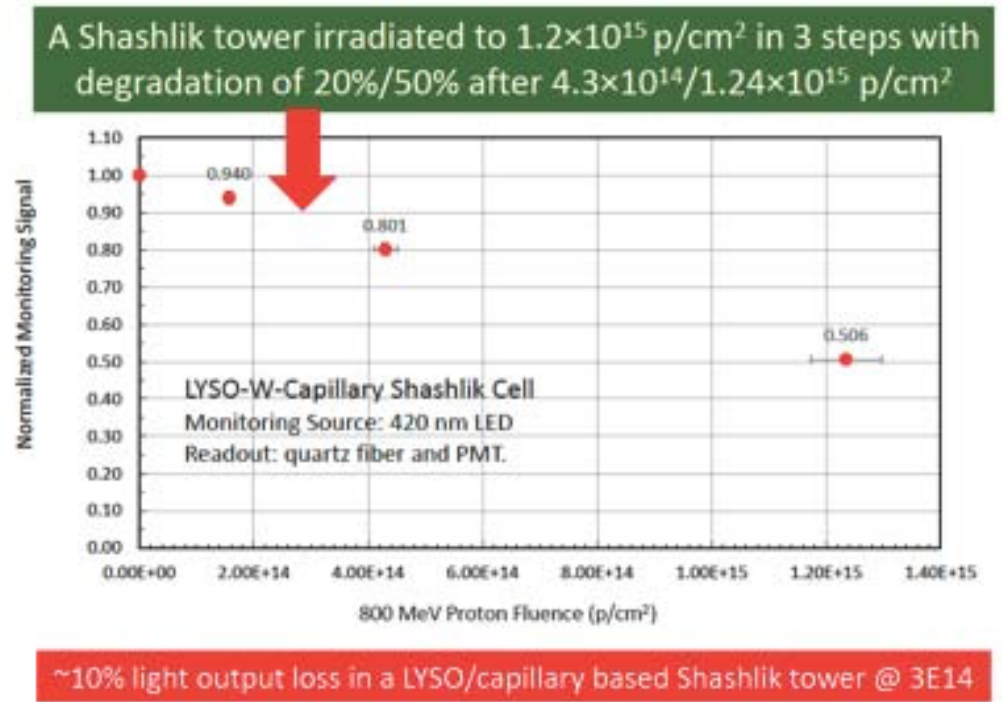

Fig. 7 Light loss due to irradiation of a Shashlik module from $4.3 \times 10-11$ to $1.2 \times 1015 \mathrm{p} / \mathrm{cm} 2$.

Finally, the vulnerability of the Shashlik structure to radiation damage has been measured by exposing a module with all the aspects of the final Shashlik design to the $800 \mathrm{MeV}$ proton beam at LANL, and measuring the light loss as a function of integrated flux. The results are shown in Fig.7 below: A factor of two light loss is experienced after exposure of up to 1015 protons per $\mathrm{cm} 2$ of integrated fluence. This is less that the expected light loss predicted by simulations of the effect of this level of fluence.

\section{Acknowledgements}

We would like to acknowledge the support of the Department of Energy, the National Science Foundation, CERN, CMS and especially US CMS and the institutions and funding agencies of the CMS Shashlik Group [5].

\section{References}

[1] “A Very Compact Crystal Shashlik Electromagnetic Calorimeter for Future HEP Experiments", R-Y Zhu, Submitted to the Proceedings of CALOR 2016, Daegu, Republic of Korea (15-20 May 2016) https://indico.cern.ch/event/472938/sessions/102269/\#2016051.

[2] “Thick-wall, Liquid-filled Quartz Capillaries for Scintillation and Wavelength Shifting Applications", B. Baumbaugh, et al, Presented by R. Ruchti and Submitted to the Proceeding of the ICHEP2016, Chicago, IL USA (4-10 August 2016)

[3] https://indico.cern.ch/event/432527/contributions/1072109/.

[4] DOE SBIR Phase I: "Radiation Hard Tight Pitch GaInP SPAD Arrays for High Energy Physics", DE-SC0013785.

[5] "Effects of very high radiation on SiPMs", A. Heering, et al, Nucl. Instr and Methods in Physics

[6] Research A 824 (2016) 111.

[7] https://www3.nd.edu/ rruchti/CMS_Shashlik_Collaboration.pdf 